\title{
Case Report: Digital analysis of occlusion with T-Scan Novus in occlusal splint treatment for a patient with bruxism [version
}

\section{1; peer review: 1 approved, 1 approved with reservations]}

\author{
Dobromira Shopova(D1, Tanya Bozhkova(iD1, Svetlana Yordanova2, \\ Miroslava Yordanova² \\ ${ }^{1}$ Department of Prosthetic Dentistry, Faculty of Dental Medicine, Medical University Plovdiv, Plovdiv, 4000, Bulgaria \\ ${ }^{2}$ Department of Orthodontics, Faculty of Dental Medicine, Medical University, Plovdiv, 4000, Bulgaria
}

V1 First published: 13 Sep 2021, 10:915
https://doi.org/10.12688/f1000research.72951.1

Latest published: 13 Jan 2022, 10:915

https://doi.org/10.12688/f1000research.72951.2

\section{Abstract}

Bruxism is a disease with a multifactorial etiology. Its clinical manifestations are most often an unaesthetic smile with abraded tooth surfaces, temporomandibular disorders and muscle hyperactivity. Here we present a case of bruxism where proper articulation of the occlusal splint was performed using the T-scan Novus system.

A patient with bruxism underwent treatment with stabilization splint made by 3D printer technology. Intraoral scanning was performed using Trios Color (3Shape, 2014), and the digital design was achieved using the 3Shape Dental system design - splint studio. Formlabs Form 2 printer with biocompatible resin Dental LT Clear Resin was used for printing. The T-Scan Novus system with software attached to it, version 9.1, was used for digital examination of the occlusion. A 2.7 $\mathrm{mm}$ thick occlusal splint was developed, and the software adapted the occlusion with antagonists. After adjustment with T-Scan Novus, a reduction in disocclusion time of the patient was achieved, which is a desired result in the treatment of bruxism. The position of the joint components was proven radiologically.

The treatment of bruxism with splint therapy continues to be the main method of treatment. Using digital technology allows for more accurate constructions and precise balancing of occlusal relationships.

Keywords

T-Scan Novus, digital splint design, 3Shape, bruxism

\section{Open Peer Review \\ Approval Status \\ 1 \\ 2 \\ version 2 \\ (revision) \\ 13 Jan 2022 \\ version 1 \\ 13 Sep 2021

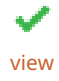 \\ a \\ ? \\ view \\ 1. Bozhidar Yordanov (D), Medical University - \\ Sofia, Sofia, Bulgaria \\ 2. Dinesh Rokaya (iD), Walailak University, \\ Bangkok, Thailand}

Any reports and responses or comments on the article can be found at the end of the article. 
Corresponding author: Dobromira Shopova (dent.shopova@gmail.com)

Author roles: Shopova D: Methodology, Project Administration, Writing - Original Draft Preparation; Bozhkova T: Formal Analysis, Visualization; Yordanova S: Supervision; Yordanova M: Writing - Review \& Editing

Competing interests: No competing interests were disclosed.

Grant information: This study was funded by Grant № 13/2020, Medical University - Plovdiv, Bulgaria.

The funders had no role in study design, data collection and analysis, decision to publish, or preparation of the manuscript.

Copyright: ( 2021 Shopova D et al. This is an open access article distributed under the terms of the Creative Commons Attribution License, which permits unrestricted use, distribution, and reproduction in any medium, provided the original work is properly cited.

How to cite this article: Shopova D, Bozhkova T, Yordanova S and Yordanova M. Case Report: Digital analysis of occlusion with TScan Novus in occlusal splint treatment for a patient with bruxism [version 1; peer review: 1 approved, 1 approved with reservations] F1000Research 2021, 10:915 https://doi.org/10.12688/f1000research.72951.1

First published: 13 Sep 2021, 10:915 https://doi.org/10.12688/f1000research.72951.1 


\section{Introduction}

Bruxism is a disease with a multifactorial etiology, such as stress, occlusal factors and trauma. Its clinical symptoms are most often abraded tooth surfaces, muscle hyperactivity, temporomandibular joint (TMJ) disorders (pain, clicking, limited opening), and in advanced cases headache and hearing problems can be observed. ${ }^{1}$

The treatment of bruxism is reduced to several main methods - increasing the vertical dimension of occlusion (VDO) to normal, medialization of the mandible to influence the position of the joint condyle in the joint fossa and release the disc, and positioning the mandible in a balanced stable occlusion. ${ }^{2,3}$ This is achieved by splint therapy for a certain adaptation period and then the result can be fixed by orthodontic treatment, adhesive restorations or prosthetic construction. ${ }^{4}$ The stabilization splint is indicated for the most common symptoms of TMJ and muscle. ${ }^{2}$

Digital technologies for dental purposes have undergone enormous development in recent years. Brand giants have developed laboratory protocols from the initial unit of digital model creation (via intraoral or laboratory scanner), through to the modeling of substructures (via design software) to the actual creation of the final product (using 3D printing and CAD/CAM methods). Modern dentistry is strongly influenced by digital technologies. ${ }^{5-7}$ Innovative technologies already have an established protocol for creating splints with personal design, from different materials and by different methods. $^{8-10}$

Achieving a balanced occlusion requires a number of evenly distributed contacts. ${ }^{11}$ Articulation paper is widely used in practice when registering occlusal contacts. The interpretation of the markers obtained with articulation paper hides the possibility of making mistakes, as it is subjective. It is wrong to perceive large and dark markers as the places with the highest load. ${ }^{12}$ With articulation paper, it is only possible to locate the contacts. The T-Scan Novus digital occlusion analysis system is used to study the sequence of occurrence and the strength of the occlusal contacts. Created 35 years ago by the Maness, this system has proven its usefulness in the study of occlusal contacts. When using T-Scan, obtained information allows accurate occlusal adjustment. The registered contacts are displayed as 2D and 3D images, and are colored in different colors depending on the applied force. The system automatically calculates occlusion time (OT) and disocussion time (DT). The OT is the elapsed time in seconds, measured from the first tooth contact until the last tooth contact. Maximum intercuspation always occurs before the patient achieves maximum bite force. The OT describes the degree of bilateral time simultaneity present in a patient occlusion. The ideal duration of OT is $\leq 0.2 \mathrm{~s}$. The DT is the elapsed time in seconds, measured from the beginning of an excursive movement made in any direction (left, right, or forward) with all teeth in maximum intercuspation until only canines and/or incisors are in contact. The ideal duration of DT is $\leq 0.5$ s. $^{13}$ Kerstein and Wright ${ }^{14}$ were the first to suggest that some patients with temporomandibular disorders, including those with bruxism, can be treated by reducing DT. They correct DT using a technique they call immediate complete anterior guidance development (ICAGD). This approach to occlusal therapy establishes immediate posterior disocclusion in all mandibular movements, prior to any habitual closure adjustments. ${ }^{15}$ Patients with myofascial pain have been shown to have increased DT. By the decreasing of DT, it reduces the hyperactivity of masseter and temporalis, the main muscles closing the lower jaw. ${ }^{15,16}$

Here, we report a patient with bruxism, where proper articulation of the occlusal splint was performed using the T-scan Novus System.

\section{Case report}

Initial presentation

A 56-year-old female patient, Bulgarian shop assistant, underwent treatment for bruxism. The leading complaints were an unaesthetic appearance of the smile, muscle fatigue almost all day and the clicking of both TMJ. Intraoral examination revealed highly abraded tooth surfaces entering the dentin area and a flattened smile line (Figures 1 and 2). The main masticatory muscles, masseter and temporalis, had increased tone, and the patient reported headaches related to this. On palpation of the TMJ, the patient responded with mild to moderate pain. There was a sharp click as the jaw was opened wide.

We decided to treat the patient with 3D printed stabilization splints. For this purpose, a digital work protocol was applied. An intraoral scan was performed with a Trios Color scanner (3Shape). The splint protocol requires imaging of the upper, lower jaw, left and right bite. The 3Shape Dental system design - splint studio was used for digital design. The splint was made using 3D printing with a Formlabs Form 2 printer and biocompatible resin (Dental LT Clear Resin).

The T-Scan Novus system with software attached to it, version 9.1, was used for digital examination of the occlusion. For the intraoral localization of the contacts, a two-stage technique was applied using Bausch 40 microns articulation paper and Bausch 12 microns articulation foil. 




Figure 1. Initial presentation showing central occlusion.

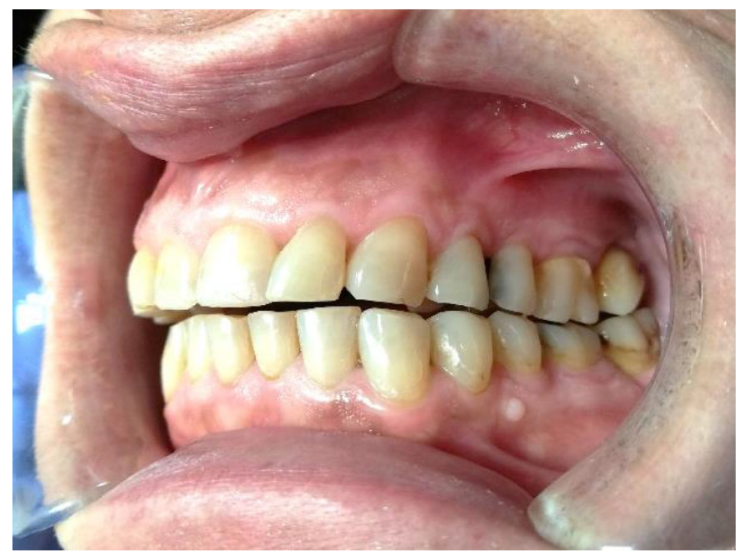

Figure 2. Initial presentation showing abraded teeth surfaces.

Initial digital design of the splint

The digital software allows different design options. In this case, the following parameters of the lower jaw position were set: vestibular thickness of the splint at $1 \mathrm{~mm}$; occlusal opening at $2.7 \mathrm{~mm}$; and protrusion at $1 \mathrm{~mm}$ (Figure 3).



Figure 3. Intermaxillary relation for splint creation (3Shape). 


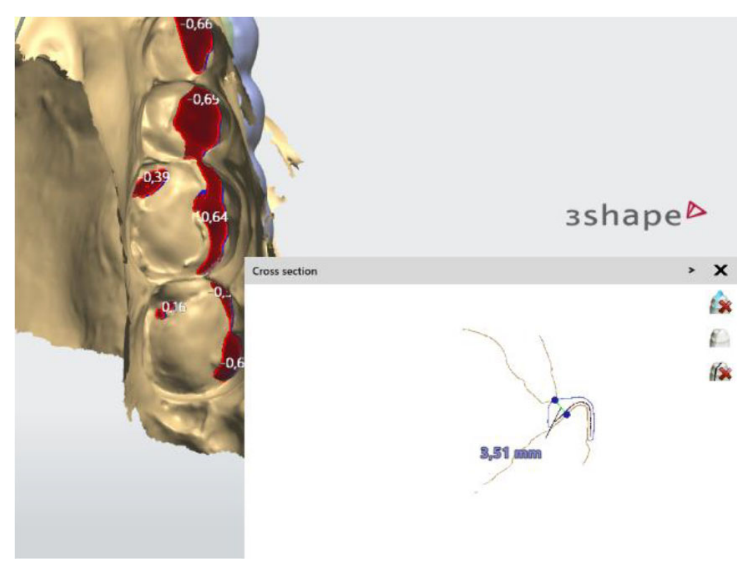

Figure 4. Frontal palatal extension of the splint (3Shape).

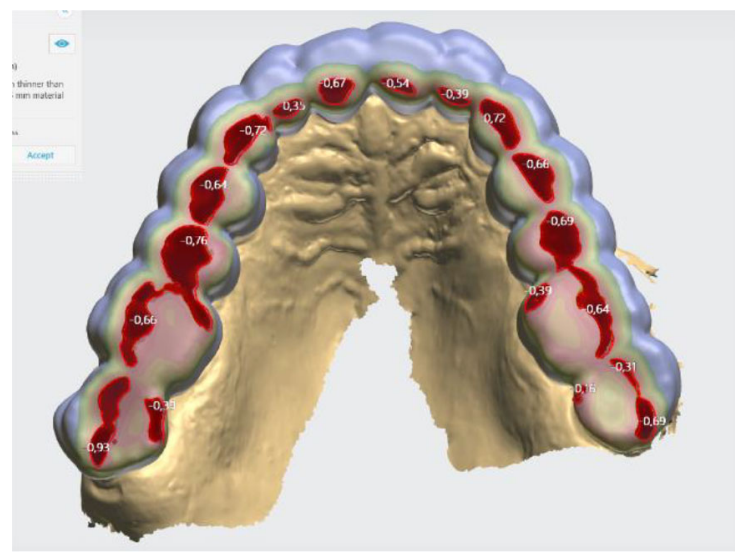

Figure 5. Balanced occlusion by the software 3Shape Splint studio.

When designing the occlusal surfaces, an option with relief to the antagonists was set. A surface modeled in this way has a slight occlusal relief, which leads the opposite jaw to the designed position. Occlusal surfaces were software-extended and provide wider contact. This is especially evident in the frontal teeth area. In this way a balanced stable occlusion was achieved. As the patient's clinical crowns were without retention areas, the splint borders were located to the cervical zone vestibularly and palatally, except the distal teeth, where the borders were $1 \mathrm{~mm}$ up of the cervix vestibularly. This extension also contributed to its stable position (Figures 4 and 5).

Once the splint had been designed, its adaptation to the tooth surfaces was checked by a silicone test (Figures 6 and 7).

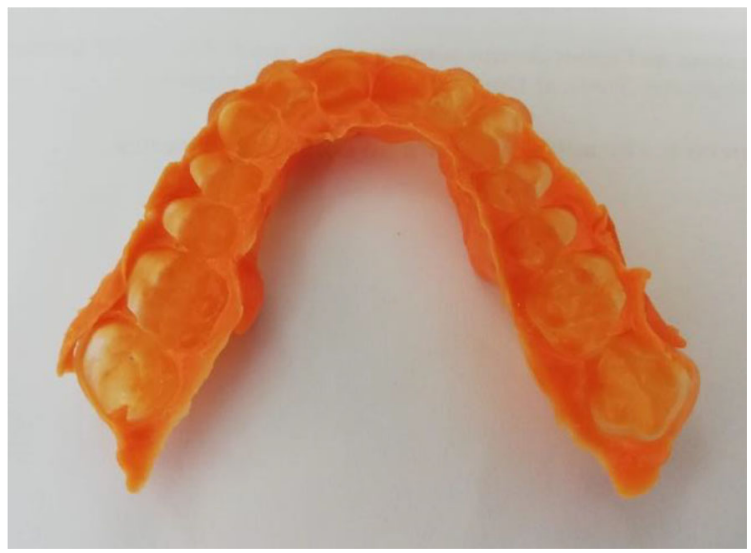




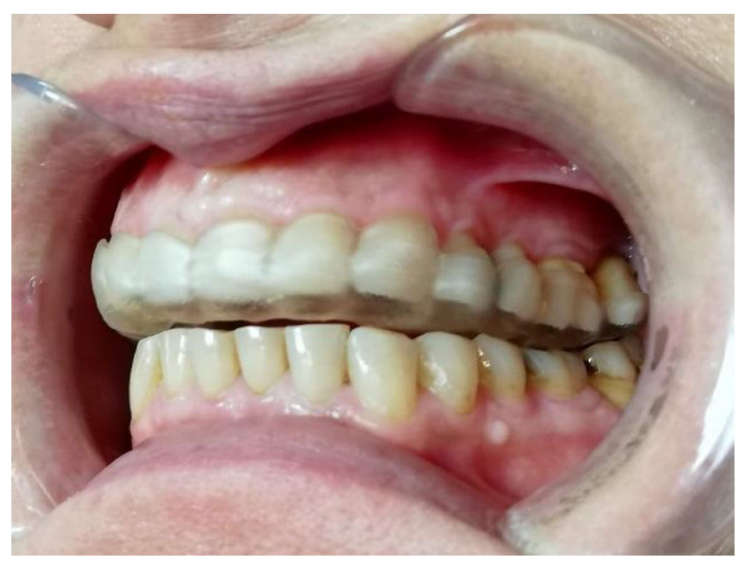

Figure 7. Stabilization splint in the patient's mouth.

\section{Computer-guided occlusal splint adjustment}

After proving its stable position on the tooth surfaces, we proceeded to computer-guided occlusal splint adjustment with the T-Scan Novus System.

Firstly, we registered occlusal contacts with the splint in the mouth using the T-Scan Novus software. Registered occlusal contacts are represented as two-dimensional contour images or three-dimensional images. The strength of the contacts is determined in color by the help of a scale; weak contacts are colored in blue and strong contacts in red (Figure 8). In our patient, uneven distribution of occlusal contacts with predominance of forces in the left side was established. In addition, center of force (COF) was shifted to the left. Elevated values of OT $(0.52 \mathrm{~s})$ and DT $(0.74 \mathrm{~s})$ were reported.

Visualization of the occlusal contacts was done intraorally with articulation paper and foil. After removing the strong contacts, a new record was made (Figure 9). This revealed that a greater load was still received by the left side, but the forces were reduced. The COF was still shifted to the left, at the periphery. Reduced OT values $(0.47 \mathrm{~s})$ were detected and DT values $(0.4 \mathrm{~s})$ were normalized.

On the last recording of the occlusion, evenly distributed contacts in the area of the lateral teeth were established. The forces on both sides are approximately equal (right side $50.3 \%$, left side $49.7 \%$ ) and COF was in the middle. The values of OT and DT were normal (0.27 s and $0.4 \mathrm{~s}$, respectively) (Figure 10).

The occlusion of the patient visualized by a two-stage technique (Bausch 40 microns articulation paper and Bausch 12 microns articulation foil) almost confirmed the result of the digitally obtained result. The frontal area was marked with contacts with the articulation paper and foil, which weren't registered by the T-Scan Novus System (Figure 11).

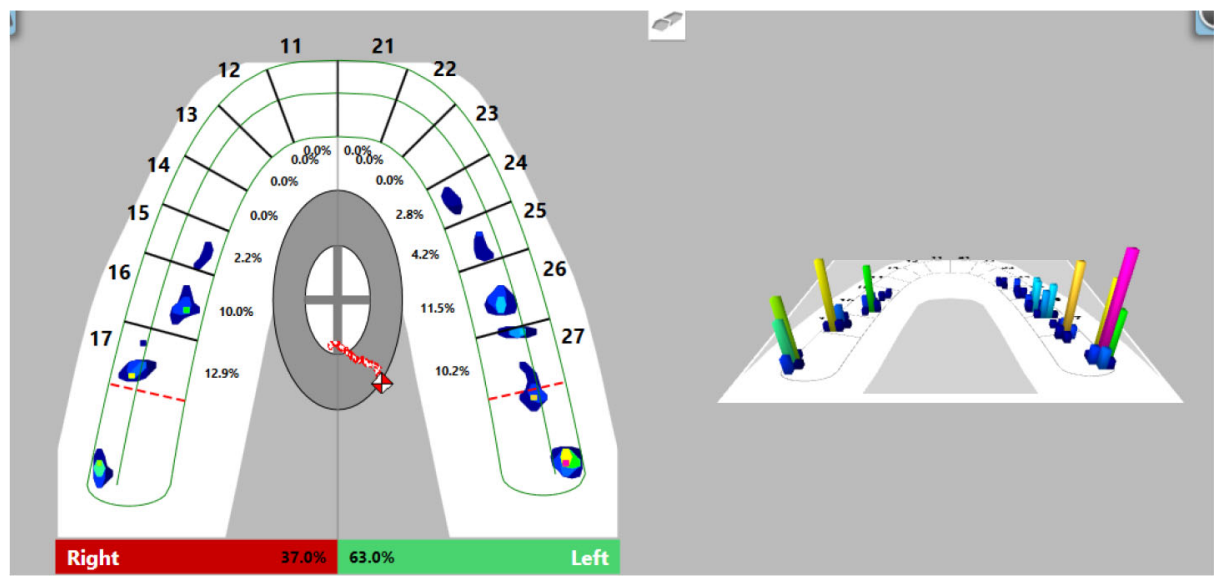

Figure 8. First occlusal registration (T-Scan Novus). 


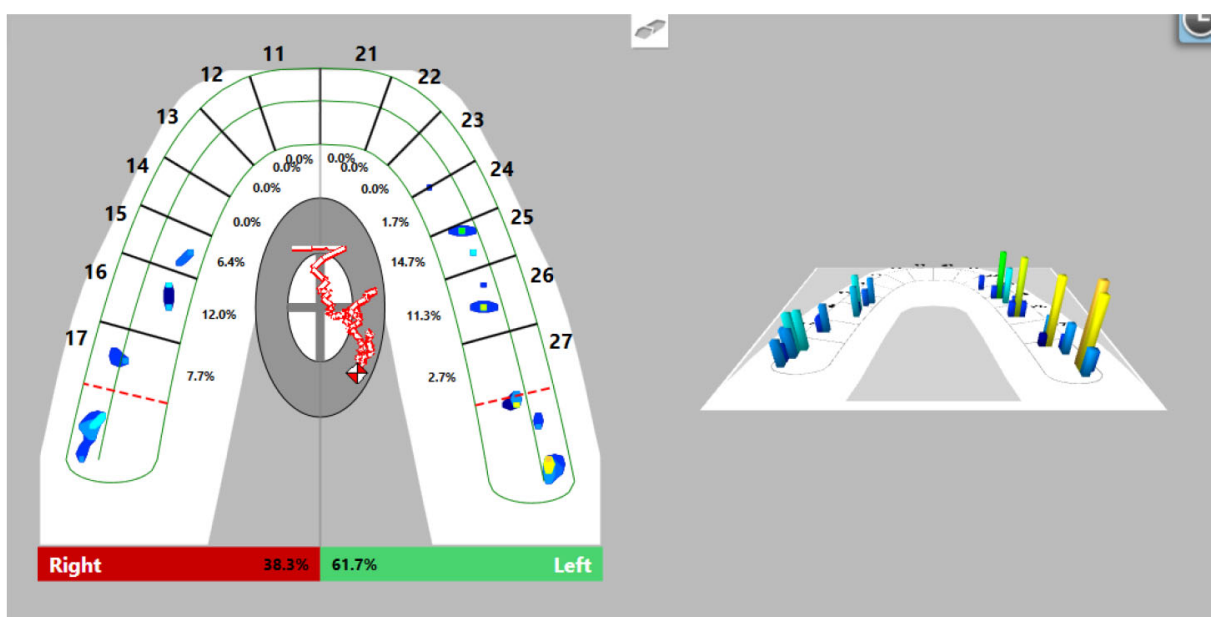

Figure 9. Intermediate occlusal registration (T-Scan Novus).

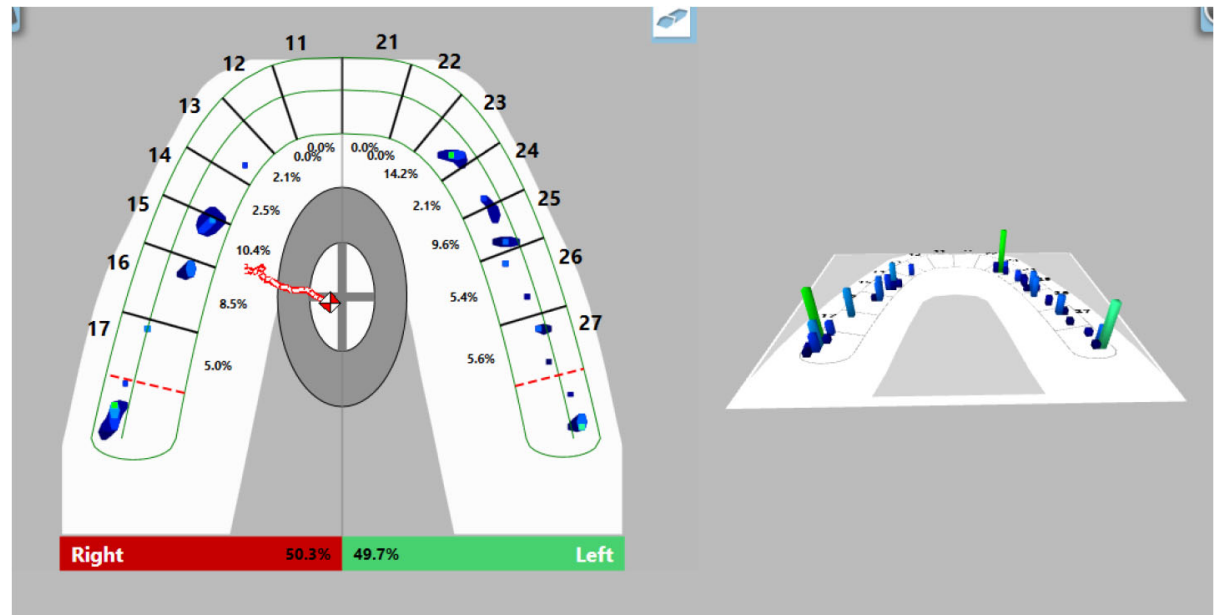

Figure 10. Final occlusal registration (T-Scan Novus).

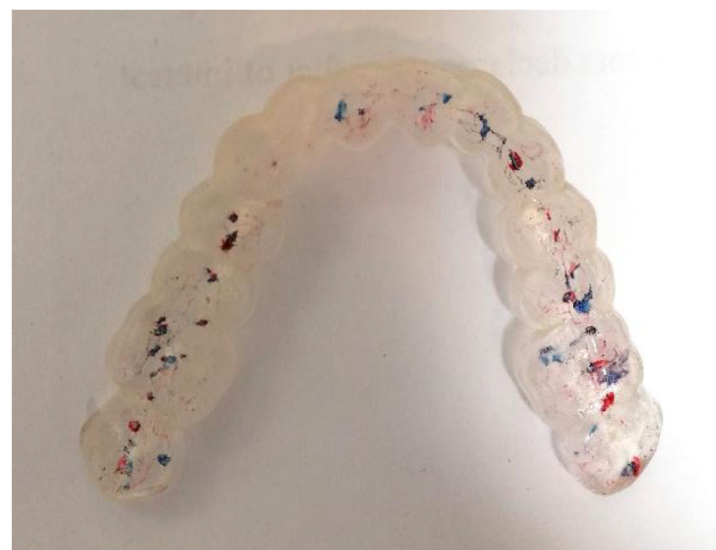

Figure 11. Occlusal registration with thin foil and articulation paper.

Follow-up

A radiological examination was performed to check the condition of the TMJ. Four positions of the joint were made: in open and closed position, with the splint, and with the splint and a $1.5 \mathrm{~mm}$ spacer (Figures 12 and 13). 

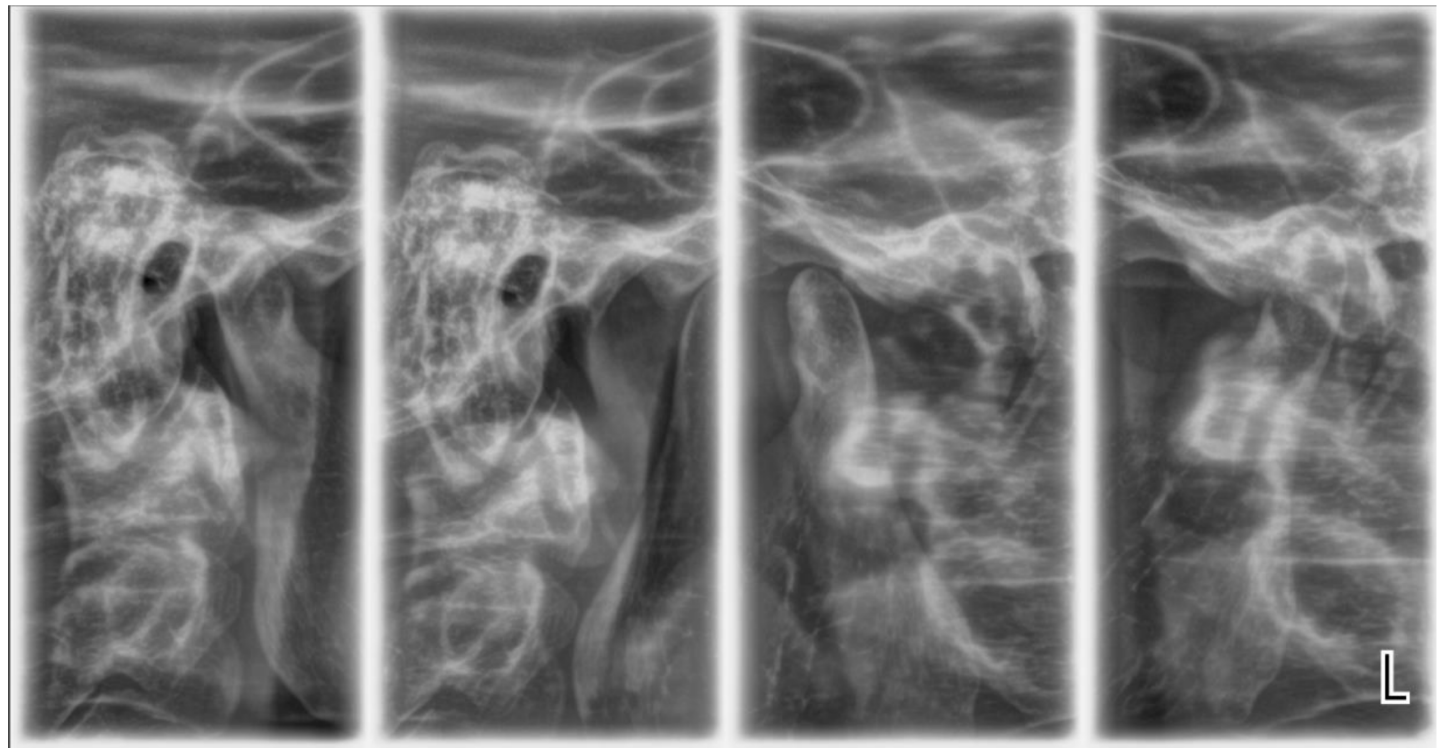

Figure 12. TMJ in open and closed position.

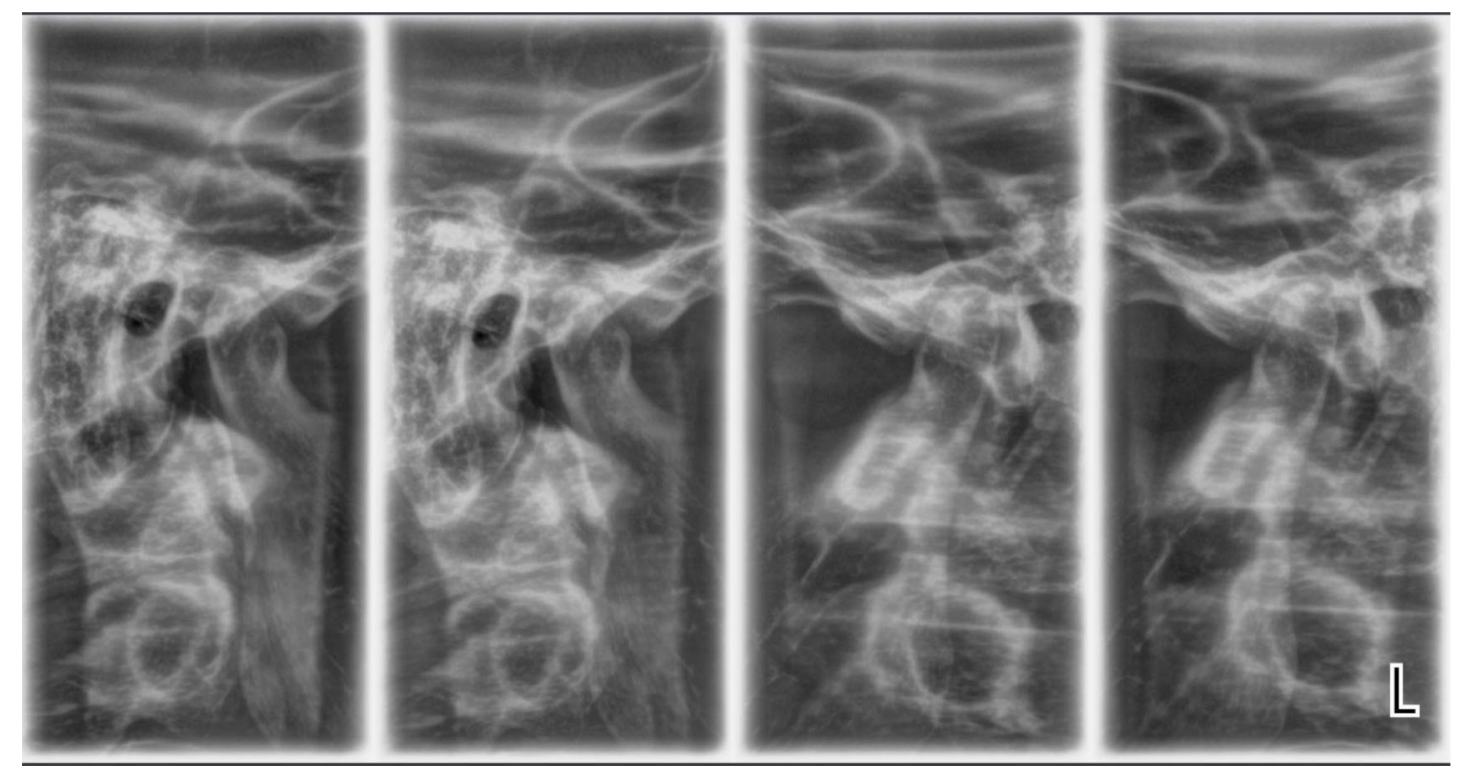

Figure 13. TMJ condition with the splint, and with the splint and spacer $1.5 \mathrm{~mm}$.

The radiological exam shows that there was symmetry between the right and left TMJ, no structural changes of the articular condyle and eminence were detected, the joint condyle was located symmetrically in the joint capsule, and when the mouth was opened, the condyle passes the eminence, the so-called habitual luxation.

\section{Discussion}

The treatment of bruxism is difficult and long. Visual evidence is needed that a treatment plan is working. T-Scan Novus visualizes the contacts between the jaws, the movement of the lower jaw, as well as establishes values of parameters (OT and DT), which are of major importance for the treatment of bruxism.

Stabilization splints for patients with bruxism aim to raise TMJ pain, muscle hyperactivity and restore VDO. ${ }^{2,3}$ Extending occlusal surfaces contributes to a more stable positioning of the lower jaw. The lack of blocks and preliminary contacts favors the final result of the treatment. ${ }^{2,4}$ 
Digital design enables a very good and even adaptation of the splint to the tooth surfaces to be achieved. Occlusal surface was less precise. The reason can be found in the minimum output thickness of the material to be made (for 3D printing), which the software automatically sets. When using a splint milling by CAD/CAM technology, the mismatch can be sought again in the minimum thickness of the material, but also the limitation of the thinnest bur for cutting. ${ }^{6-8}$

The T-Scan Novus System shows more than just occlusal contacts. In the case of bruxism, it is more valuable to rely on OT and DT. In our study, during the clinical adjustment, a reduction of the values was achieved by almost half; the reported OT and DT approached the norm (OT: $0.27 \mathrm{~s}$, norm $\leq 0.2 \mathrm{~s}$; DT: $0.4 \mathrm{~s}$, norm $\leq 0.5 \mathrm{~s}$ ). The achieved result (DT) is a proven method of treatment for bruxism. ${ }^{14,15}$

In our study, visualization of the registered intraoral occlusal contacts was performed by a two-stage technique with articulation paper 40 microns and articulation foil 12 microns. When comparing the obtained results with those of the T-Scan Novus system, differences in the number and location of the occlusal contacts were established. This proves the possibility of registering false positive contacts when using articulation paper. ${ }^{12}$

Radiological examination is the only option for objective examination of TMJ. As an initial study it serves to analyze the anatomical structures, their location relative to each other, and to exclude other causes of joint pain (trauma, ankylosis, tumor disease). X-rays taken after splinting prove the position of the joint components. In case of unsatisfactory or irrelevant placement, the splint should be redesigned. ${ }^{1,4}$

\section{Conclusion}

The main method of bruxism treatment continues to be splint therapy. Its combination with digital technologies allows more precise constructions and more detailed visualizations at each stage - design and dental adaptation of the splint, occlusal relief and occlusal relationships. As shown in our patient, with the T-Scan Novus system, it is possible to achieve optimal, harmonious occlusal ratios.

\section{Consent}

Written informed consent was obtained from the patient for the publication of the case report with any associated images.

\section{Data availability}

All data underlying the results are available as part of the article and no additional source data are required.

\section{Acknowledgements}

Intraoral scanning and splint design were made at the CAD/CAM Center Dental Medicine at the Research Institute, Medical University - Plovdiv, Bulgaria.

\section{References}

1. Okeson JP: Management of Temporomandibular Disorders and Occlusion-E-Book. Elsevier Health Sciences. 2019.

2. Al-Ani M, Ziad, et al.: Stabilisation splint therapy for temporomandibular pain dysfunction syndrome. Cochrane Database of Systematic Reviews. 2004; 1 PubMed Abstract | Publisher Full Text

3. Lakshmi MS, et al.: Occlusal Splint Therapy in Temporomandibular Joint Disorders: An Update Review. J International Oral Health. 2016; 8(5). Publisher Full Text | Free Full Text

4. Bumann A, Lotzmann U: TMJ Disorders and Orofacial Pain. Stuttgart, New York: Thieme; 2002.

5. Dawood A, Marti BM, Sauret-Jackson V, et al.: 3D printing in dentistry. Br Dental J. 2015; 219(11): 521-529. Publisher Full Text

6. Mikolajczyk T, Malinowski T, Moldovan L, et al.: CAD CAM system for manufacturing innovative hybrid design using 3D printing. Procedia Manufacturing. 2019; 32: 22-28. Publisher Full Text

7. Zhou Q, Wang Z, Chen J, et al.: Development and evaluation of a digital dental modeling method based on grating projection and reverse engineering software. J Prosthetic Dentistry. 2016;
115(1): 42-46.

Publisher Full Text

8. Edelhoff $D$, et al.: CAD/CAM splints for the functional and esthetic evaluation of newly defined occlusal dimensions. Quintessence Int. 2017; 48.3.

9. Nota A, Ryakhovsky AN, Bosco F, et al.: A Full Digital Workflow to Design and Mill a Splint for a Patient with Temporomandibular Joint Disorder. Appl. Sci. 2021; 11(1): 372. Publisher Full Text

10. Venezia $P$, Muzio LL, De Furia $C$, et al.: Digital manufacturing of occlusal splint: From intraoral scanning to 3D printing. J Osseointegration. 2019; 11(4): 535-539. Publisher Full Text

11. Atashrazm P, Lari HA, Khorsand M: An Evaluation of Occlusal Contacts of Remounted Complete Denture before Final Occlusal Adjustment. J Dentistry. Shiraz University of Medical Sciences. 2009; 9: 1-5.

12. Carey J, Craig M, Kerstein RB, et al.: Determining a relationship between applied occlusal load and articulation paper mark area. Open Dent J. 2007; 1: 1-7.

PubMed Abstract | Publisher Full Text | Free Full Text 
13. Kerstein R: Handbook of research on computerized occlusal analysis technology applications in dental medicine. IGI global. 2014: 1-15.

14. Kerstein RB, Wright NR: Electromyographic and computer analyses of patients suffering from chronic myofascial paindysfunction syndrome: before and after treatment with immediate complete anterior guidance development. J Prosthet Dent. 1991; 66: 677-686.

PubMed Abstract | Publisher Full Text

15. Kerstein RB: Disocclusion time-reduction therapy with immediate complete anterior guidance development to treat chronic myofascial pain-dysfunction syndrome. Quintessence Int
1992; 23: 735-747 PubMed Abstract

16. Kerstein RB, Lowe $\mathrm{M}$, Harty $\mathrm{M}$, et al.: A force reproduction analysis of two recording sensors of a computerized occlusal analysis system. Cranio. 2006; 24: 15-24.

PubMed Abstract | Publisher Full Text

17. Kerstein RB: Reducing chronic masseter and temporalis muscular hyperactivity with computer-guided occlusal adjustments. Compend Contin Educ Dent. 2010; 31:

530-534. 536538

PubMed Abstract 


\section{Open Peer Review}

\section{Current Peer Review Status:}

\section{Version 1}

Reviewer Report 18 November 2021

https://doi.org/10.5256/f1000research.76564.r99206

(C) 2021 Rokaya D. This is an open access peer review report distributed under the terms of the Creative Commons Attribution License, which permits unrestricted use, distribution, and reproduction in any medium, provided the original work is properly cited.

\section{Dinesh Rokaya}

Department of Clinical Dentistry, Walailak University International College of Dentistry, Walailak University, Bangkok, Thailand

This article presents the management of bruxism with occlusal splint with adjustment done using the T-scan Novus system.

\section{Abstract: \\ Please mention which Trios was used, 3 or 4. \\ Introduction: \\ At present, although various scanners are available in the market, the Trios system presents the best scan which system is used in this case study. \\ The authors need to add the following current literature in the introduction: \\ Amornvit et al., $2021^{1}$ - This study compared the various intraoral scanners and the results show that Trios showed the best results. \\ Amornvit et al., $2020^{2}$ - This study explained the confocal technique used in Trios which showed high accuracy especially in the posterior region.}

\section{Case Report:}

It is better to add details of the product and software such as Trios, resin, etc. Product name, Company, City, and Country.

Figures 12 and 13: Each X-ray needs to be labeled.

How often the recall was done and how long the splint was given needs to be mentioned.

\section{Discussion:}

At present, scanning the upper arch, lower arch and bite allows to check the occlusion and force on the area of each tooth using 3Shape Dental System. This is also shown in this case. The occlusion can also be done more precisely using T-Scan but the disadvantage is this will add extra cost to the dentist and the patient. Hence, the authors need to add the specific 
use of T-Scan in this case study.

In addition, the authors can discuss the indications and limitations of each system in checking the occlusion. This comparison can be done using a table. The following articles can be helpful: Lee et al., 201833; Cheng et al., 20184; Solaberrieta et al., $2016^{5}$.

\section{References}

1. Amornvit P, Rokaya D, Sanohkan S: Comparison of Accuracy of Current Ten Intraoral Scanners. Biomed Res Int. 2021; 2021: 2673040 PubMed Abstract | Publisher Full Text 2. Amornvit P, Rokaya D, Peampring C, Sanohkan S: Confocal 3D Optical Intraoral Scanners and Comparison of Image Capturing Accuracy. Computers, Materials \& Continua. 2020; 66 (1): 303-314 Publisher Full Text

3. Lee $\mathrm{H}$, Cha J, Chun Y, Kim M: Comparison of the occlusal contact area of virtual models and actual models: a comparative in vitro study on Class I and Class II malocclusion models. BMC Oral Health. 2018; 18 (1). Publisher Full Text

4. Cheng MX, Jiang T, Sun YC, Zhang HY: [Influence of intraoral scan and dental cast scan on occlusal quantitative analysis of virtual dental model].Beijing Da Xue Xue Bao Yi Xue Ban. 2018; 50

(1): 136-140 PubMed Abstract

5. Solaberrieta E, Garmendia A, Brizuela A, Otegi JR, et al.: Intraoral Digital Impressions for Virtual Occlusal Records: Section Quantity and Dimensions.Biomed Res Int. 2016; 2016: 7173824 PubMed Abstract | Publisher Full Text

Is the background of the case's history and progression described in sufficient detail? Yes

Are enough details provided of any physical examination and diagnostic tests, treatment given and outcomes?

Yes

Is sufficient discussion included of the importance of the findings and their relevance to future understanding of disease processes, diagnosis or treatment?

Partly

Is the case presented with sufficient detail to be useful for other practitioners? Partly

Competing Interests: No competing interests were disclosed.

Reviewer Expertise: Clinical dentistry, prosthetic dentistry, digital dentistry, dental biomaterials

I confirm that I have read this submission and believe that I have an appropriate level of expertise to confirm that it is of an acceptable scientific standard, however I have significant reservations, as outlined above. 
dobromira shopova, Medical University Plovdiv, Plovdiv, Bulgaria

Dear Reviewer,

Thank you for your advices! I will answer point by point:

Abstract:
$\quad$ Please mention which Trios was used, 3 or 4.

Author response: The previous generation intraoral scanner of 3Shape Trios color, from 2014, was used.

\section{Introduction:}

At present, although various scanners are available in the market, the Trios system presents the best scan which system is used in this case study.

The authors need to add the following current literature in the introduction:

Amornvit et al., $2021^{1}$ - This study compared the various intraoral scanners and the results show that Trios showed the best results.

Amornvit et al., $2020^{2}$ - This study explained the confocal technique used in Trios which showed high accuracy especially in the posterior region.

Author response: I added them, thank you!

\section{Case Report:}

It is better to add details of the product and software such as Trios, resin, etc. Product name, Company, City, and Country.

Author response: The information is added!

Figures 12 and 13: Each X-ray needs to be labeled.

Author response: I labeled them, thank you!

How often the recall was done and how long the splint was given needs to be mentioned.

Author response: After 3 months the recall was made. Clinical symptoms were reduced. The control study with T-Scan showed small changes, but within the same limits of OT and DT as the placement.

\section{Discussion:}

At present, scanning the upper arch, lower arch and bite allows to check the occlusion and force on the area of each tooth using 3Shape Dental System. This is also shown in this case. The occlusion can also be done more precisely using T-Scan but the disadvantage is this will add extra cost to the dentist and the patient. Hence, the authors need to add the specific use of T-Scan in this case study.

Author response: Our study with the T-Scan system revealed an unbalanced distribution of occlusal contacts, although they were designed to be balanced by 3Shape Splint studio software. The reason can be found in the minimum output thickness of the material to be made (for 3D printing), and which the software automatically sets. 
There is evidence in the literature that one of the limitations of intraoral scanners is that they cannot measure the intensity of occlusal forces. The T-Scan system has been shown to be a reliable method for assessing the occlusal contact zone (Ayuso-Montero, R., MarianoHernandez, Y., Khoury-Ribas, L., Rovira-Lastra, B., Willaert, E., \& Martinez-Gomis, J. (2020). Reliability and validity of T-scan and 3d intraoral scanning for measuring the occlusal contact area. Journal of Prosthodontics, 29(1), 19-25).

In addition, the authors can discuss the indications and limitations of each system in checking the occlusion. This comparison can be done using a table. The following articles can be helpful: Lee et al., 20183; Cheng et al., 20184'; Solaberrieta et al., $2016^{5}$. Author response: The use of the T-Scan system provides data for more in-depth occlusal analysis. Through this study, it is possible to determine whether the occlusion is balanced and also the sequence, strength, and location of the occlusal contacts. 3Shape software does not have these features. Our opinion on the benefits of using the computerized analysis system is confirmed by other authors (Kerstein RB. Current applications of computerized occlusal analysis in dental medicine. Gen Dent. 2001; 49: 521-530; Buduru S, Mesaros A, Talmaceanu D., Baru O, Ghiurca R, Cosgarea R. Occlusion in the digital era: a report on 3 cases. Medicine and pharmacy reports. 2019; 92 (3): 78). Therefore, we recommend that, if technically possible, you should not rely solely on the information provided by the intraoral scanner, however accurate it may be.

Competing Interests: No competing interests were disclosed.

Reviewer Report 21 October 2021

https://doi.org/10.5256/f1000research.76564.r95636

(C) 2021 Yordanov B. This is an open access peer review report distributed under the terms of the Creative Commons Attribution License, which permits unrestricted use, distribution, and reproduction in any medium, provided the original work is properly cited.

\section{Bozhidar Yordanov}

Department of Prosthetic Dental Medicine, Faculty of Dental Medicine, Medical University - Sofia, Sofia, Bulgaria

The currentness of the subject discussed in this paper is determined by the contemporary requirements for optimum rehabilitation of the masticatory system in patients with bruxism. Splint therapy of bruxism is still the basic method of treatment to relieve specific clinical symptoms - temporomandibular disorders, muscle hyperactivity, and lost esthetics as a result of abrasion of tooth occlusal surfaces. Contemporary digital methods for the examination of occlusion and fabrication of occlusal splints contribute to the precision and better final results following occlusal therapy. Patient examination, treatment planning, digital process for fabrication of the splint, adjustment of occlusal contacts using conventional and modern methods and technologies, discussion of the results and conclusions are sufficient enough for understanding diagnosis, planning, treatment of bruxism and to be useful for dental practitioners. 
Critical notes:

1. The background of the case's history and progression is not completely described in sufficient detail.

2. The scientific value of this paper will be increased by replacing some specific terms with more accurate ones: occlusal splint instead of stabilization splint; dental medicine instead of dentistry; bilaterally balanced occlusion instead of balanced occlusion; adjustment of occlusal contacts instead of articulation; retail worker instead of shop assistant; opposing teeth instead of antagonists; anterior instead of frontal; occlusal interferences instead of blocks.

3. In the second section of the Discussion, probably there is a mistake: "Stabilization splints for patients with bruxism aim to RAISE TMJ pain, muscle hyperactivity...". Splints are used to relieve pain, not to raise it.

The critical notes above do not decrease the scientific and practical value of the presented article.

Is the background of the case's history and progression described in sufficient detail? Partly

Are enough details provided of any physical examination and diagnostic tests, treatment given and outcomes?

Yes

Is sufficient discussion included of the importance of the findings and their relevance to future understanding of disease processes, diagnosis or treatment?

Yes

Is the case presented with sufficient detail to be useful for other practitioners? Yes

Competing Interests: No competing interests were disclosed.

Reviewer Expertise: Prosthetic Dental Medicine and Dental Implantology

I confirm that I have read this submission and believe that I have an appropriate level of expertise to confirm that it is of an acceptable scientific standard.

Author Response 02 Dec 2021

dobromira shopova, Medical University Plovdiv, Plovdiv, Bulgaria

Dear Reviewer,

Thank you for your advices! I will correct them in the final version of the article!

Competing Interests: No competing interests were disclosed. 
The benefits of publishing with F1000Research:

- Your article is published within days, with no editorial bias

- You can publish traditional articles, null/negative results, case reports, data notes and more

- The peer review process is transparent and collaborative

- Your article is indexed in PubMed after passing peer review

- Dedicated customer support at every stage

For pre-submission enquiries, contact research@f1000.com 